\title{
On the nature of optical emission from radio pulsars
}

\author{
I.F.Malov \\ Pushchino Radio Astronomy Observatory, Lebedev Physical Institute, \\ 142092, Pushchino, Russia

\section{G.Z.Machabeli} \\ Abastumani Astrophysical Observatory, 380060, Tbilisi, Al.Kazbegi ave., \\ 2a, Georgia
}

There are more than one thousand known radio pulsars, but only 9 of them have detected optical emission (Caraveo 1999). A part of optical emission can be caused by thermal radiation from the hot surface of the neutron star. We shall try to describe the non-thermal component (Kurt et al. 1980) on the base of the synchrotron mechanism, using one-dimensional distribution function of emitting electrons.

One of the possible reasons of observed pulsar emission is the cyclotron instability developed in an anisotropic plasma (Sagdeev \& Shafranov 1960). To generate transversal ( $\mathrm{t}$ ) waves with the spectrum

$$
\begin{gathered}
\omega_{t}=k c(1-\delta), \\
\delta=\frac{\omega_{p}^{2}}{4 \omega_{B}^{2} \gamma_{p}^{3}}, \quad \omega_{p}^{2}=\frac{4 \pi n_{p} e^{2}}{m}, \quad \omega_{B}=\frac{e B}{m c} .
\end{gathered}
$$

the condition of the cyclotron resonance (Kazbegi et al. 1992)

$$
\frac{k_{\perp}^{2}}{2 k_{\|}^{2}}+\frac{1}{2 \gamma_{r}^{2}}-\frac{k_{\perp} u_{\perp}}{k_{\|} c}-\delta= \pm \frac{\omega_{B}}{\gamma_{r} k_{\|} c}
$$

must be fulfilled. Here $k_{\|}^{2}+k_{\perp}^{2}=k^{2}, \gamma_{r}$ is the Lorentz-factor of resonance particles, $u_{1}=\frac{c V_{\|} \gamma}{\rho \omega_{B}}$ is the drift velocity of particles, $\rho$ is the curvature radius of field lines.

Plus in this equation corresponds to the excitation of waves, minus to their absorption. Both these processes lead to a redistribution of particles due to the quasilinear diffusion.

The equation (3) can be written approximately as

$$
\delta=\frac{\omega_{B}}{\omega \gamma_{r}} .
$$

and we have the next estimate of the level of the wave excitation

$$
\frac{r}{R_{*}}=\left(2 \cdot 10^{39} \frac{\gamma_{p}^{4} P B_{12}^{2}}{\gamma_{b}^{2} \omega}\right)^{1 / 6}
$$


If we put $\gamma_{p} \sim 10$ and $\gamma_{b} \sim 10^{6}$ then waves are excited near the light cylinder with $r_{L C}=\frac{c P}{2 \pi}$.

The kinetic equation for the distribution function of beam particles can be written in this case as

$$
\frac{1}{m c \gamma_{b}^{2} \psi} \frac{d}{d \psi}\left(\psi G_{\perp} f^{\circ}\right)=\frac{1}{m^{2} c^{2} \gamma_{b}^{2}} \frac{1}{\psi} \frac{d}{d \psi}\left(\psi D_{\perp \perp} \frac{d f^{\circ}}{d \psi}\right)
$$

This equation has the solution

$$
\chi(\psi)=C e^{\int \frac{G_{\perp} m c \gamma_{b}}{D_{\perp \perp}} d \psi}=C E^{-A \psi^{2}}, \quad A=\frac{2 m^{2} c^{4} \gamma_{b}^{2}\left(\frac{\omega_{B}}{\omega_{p}}\right)^{2}}{\pi e^{2} \rho|E(k)|^{2} \gamma_{p}}
$$

where

$G_{\perp}=-\frac{m c^{2}}{\rho} \gamma_{r} \psi, G_{\|}=\frac{m c^{2}}{\rho} \gamma_{r} \psi^{2}, D_{\perp \perp} \approx \frac{\pi e^{2} \omega_{p}^{2}}{4 c \omega_{B}^{2}} \gamma_{p}|E(k)|_{k=k}^{2},|E(k)|^{2} \approx \frac{12 \pi m^{2} c^{7} \gamma_{b} n_{b}}{e^{2} r \omega_{B}^{3}}$

The mean value of pitch-angle is

$$
\bar{\psi}=\left[\frac{24 \pi^{2} m^{4} c^{6} \rho\left(r / R_{*}\right)^{9}}{e^{5} B_{s}^{3} P^{2} r}\right]^{1 / 2}
$$

For PSR $0656+14$ with $r / r_{L C}=0.5 \bar{\psi}=3.6 \cdot 10^{-4}$ in the rest plasma frame.

$$
\psi_{H} \approx \frac{\bar{\psi}}{2 \gamma_{p}},=1.8 \cdot 10^{-5}
$$

in the observer's frame for $\gamma_{p}=10$. If $\gamma_{b}=10^{5}$ the frequency of the maximum in the synchrotron spectrum (Epstein 1973)

$$
\nu_{m}=a(\gamma \psi) \frac{e B}{2 \pi m c \gamma \psi^{2}}
$$

is of $10^{15} \mathrm{~Hz}$ for PSR $0656+14$. The intensity decreases slowly after the maximum to the higher frequencies. Such behaviour is in an agreement with the observed optical spectrum of PSR0656+14 in the frequency range from $3 \cdot 10^{14}$ $\mathrm{Hz}$ to $8.9 \cdot 10^{14} \mathrm{~Hz}$.

Acknowledgments. This work was supported by the INTAS grant (no.960154) and by the Russian Foundation for Basic Researches (project no.97-0217372).

\section{References}

Caraveo P.A. these proceedings

Kurt V.G., Sokolov V.V., Zharikov S.A. et al. 1980, A\&A301, 547

Sagdeev R.Z., Shafranov V.D. 1960, Zh. Exp.Theor.Phys., 39, 181

Kazbegi A.Z.,Machabeli G.Z.,Melikidze G.I. Proc IAU Colloq. 128. 1992. P.232.

Epstein R.I. 1973, ApJ183, 593 